\title{
Direct visualisation of collateral ventilation in COPD with hyperpolarised gas MRI
}

\author{
Helen Marshall, ${ }^{1}$ Martin H Deppe, ${ }^{1}$ Juan Parra-Robles, ${ }^{1}$ Susan Hillis, ${ }^{2}$ \\ Catherine G Billings, ${ }^{2}$ Smitha Rajaram, ${ }^{1}$ Andrew Swift, ${ }^{1}$ Sam R Miller, ${ }^{3}$ \\ Joanna H Watson, ${ }^{3}$ Jan Wolber, ${ }^{4}$ David A Lipson, ${ }^{5}$ Rod Lawson, ${ }^{2}$ Jim M Wild ${ }^{1}$
}

'Department of Academic Radiology, University of Sheffield, Sheffield, South Yorkshire, UK

${ }^{2}$ Department of Respiratory Medicine, Sheffield Teaching Hospitals NHS Trust, South Yorkshire, UK

${ }^{3}$ GlaxoSmithKline, Stockley Park, UK

${ }^{4}$ GE Healthcare, Amersham, UK

${ }^{5}$ GlaxoSmithKline, King of

Prussia, Pennsylvania, USA

Correspondence to Professor Jim M Wild, Department of Academic Radiology, C Floor, Royal Hallamshire Hospital, Glossop Road, Sheffield S10 2JF, UK; j.m.wild@sheffield.ac.uk

Received 27 July 2011 Accepted 8 January 2012 Published Online First 27 January 2012

\section{ABSTRACT}

Background Collateral ventilation has been proposed as a mechanism of compensation of respiratory function in obstructive lung diseases but observations of it in vivo are limited. The assessment of collateral ventilation with an imaging technique might help to gain insight into lung physiology and assist the planning of new bronchoscopic techniques for treating emphysema.

Objective To obtain images of delayed ventilation that might be related to collateral ventilation over the period of a single breath-hold in patients with chronic obstructive pulmonary disease (COPD).

Methods Time-resolved breath-hold hyperpolarised ${ }^{3} \mathrm{He}$ MRI was used to obtain images of the progressive influx of polarised gas into initially non-ventilated defects.

Results $\mathrm{A}$ time-series of images showed that ${ }^{3} \mathrm{He}$ moves into lung regions which were initially non-ventilated. Ventilation defects with delayed filling were observed in 8 of the 10 patients scanned. Conclusions A method for direct imaging of delayed ventilation within a single breath-hold has been demonstrated in patients with COPD. Images of what is believed to be collateral ventilation and slow filling of peripheral airspaces due to increased flow resistance are presented. The technique provides 3D whole-lung coverage with sensitivity to regional information, and is non-invasive and non-ionising.

\section{INTRODUCTION}

Collateral ventilation pathways have been proposed as a mechanism of increased tolerance to obstructive lung diseases by enabling alternative respiratory pathways to carry out gas exchange, ${ }^{1} 2$ see figure 1. In addition to gaining insight into lung physiology, the assessment of collateral ventilation with an imaging technique might help with the planning of new bronchoscopic techniques for treating emphysema. ${ }^{2} 3$ In bronchoscopic lung volume reduction, collateral ventilation from adjacent lobes prevents the target atelectasis, ${ }^{4}$ whereas in airway bypass treatment the presence of collateral ventilation can be advantageous. ${ }^{5}$

Catheter-based techniques ${ }^{6}$ have been used to measure collateral ventilation but a non-invasive method is desirable. Radiological CT scores of emphysema are significantly linked to interlobar collateral ventilation, ${ }^{7}$ and ${ }^{133} \mathrm{Xe}$ scintigraphy ${ }^{8}$ and xenon-enhanced dynamic dual-energy $\mathrm{CT}^{9-11}$ have been used to obtain images of collateral ventilation. However, these techniques monitor the wash-in

\section{Key messages}

What is the key question?

- Is it possible to visualise collateral ventilation in chronic obstructive pulmonary disease (COPD) during a single breath-hold using a non-invasive, non-ionising imaging technique?

What is the bottom line?

- We demonstrate direct imaging of delayed gas filling in what we believe to be collateral ventilation in patients with COPD, using hyperpolarised gas MRI.

\section{Why read on?}

- The ability to image and quantify collateral ventilation pathways directly may increase the understanding of pathophysiology in COPD and aid assessment for treatments.

and wash-out of a tracer gas over multiple breathing cycles, and both use ionising radiation. Long-range diffusion measurements with hyperpolarised ${ }^{3} \mathrm{He} \mathrm{MRI}$ are also potentially sensitive to collateral ventilation. ${ }^{12-14}$ Lung structure at the acinar level influences the long-range apparent diffusion coefficient (ADC), which is related to the path of gas through the peripheral airways. High long-range $\mathrm{ADC}$ values suggest the presence of collateral pathways because the gas has diffused more quickly than it could have done by navigating healthy airways. ${ }^{13}$ However, the measurement of long-range $\mathrm{ADC}$ is indirect and as such can only imply that collateral ventilation is taking place.

Here we present direct visual evidence of delayed gas ventilation at breath-hold in what we believe to be collateral ventilation in chronic obstructive pulmonary disease (COPD). Images were acquired over the period of a single breath-hold using a noninvasive and non-ionising imaging modality. Delayed filling of peripheral regions was also seen in some patients, which is thought to be due to increased resistance in the small airways. Timeresolved hyperpolarised ${ }^{3} \mathrm{He}$ MRI was used to image delayed ventilation in patients with COPD with whole-lung coverage.

\section{METHODS}

Ten patients with COPD were scanned using a $1.5 \mathrm{~T}$ whole-body MRI system (GE HDx, 


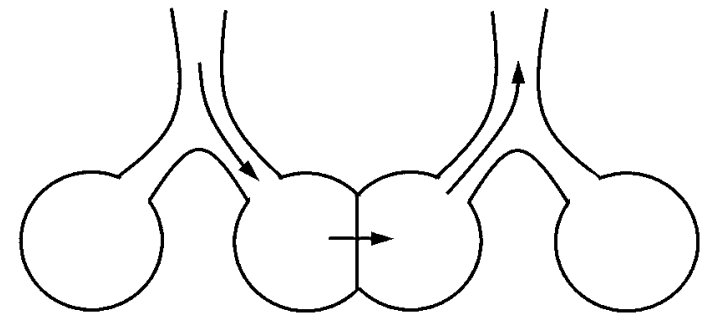

Figure 1 A graphical representation of collateral ventilation, adapted from Hogg et al. ${ }^{1}$

Milwaukee, Wisconsin, USA) equipped for hyperpolarised ${ }^{3} \mathrm{He}$ imaging. The inclusion criteria were COPD without other significant respiratory disease as diagnosed by a respiratory physician, post-bronchodilator ratio of forced expiratory volume in $1 \mathrm{~s}$ (FEV1) to forced vital capacity $<0.7$, post-bronchodilator FEV $1 \geq 30 \%$ and $\leq 80 \%$ of predicted, cigarette smoking history of $\geq 10$ pack-years and resting pulse oximeter oxygen saturation of $>90 \%$ on room air.

${ }^{3} \mathrm{He}$ was polarised on site to $25 \%$ using rubidium spinexchange $^{15}$ apparatus (GE Healthcare, Amersham, UK) under a regulatory approved licence. Imaging was performed with ethics committee approval and written consent. Subjects were positioned in a ${ }^{3} \mathrm{He}$ quadrature transmit-receive vest coil (Clinical MR Solutions, Brookfield, WI, USA). A mix of $200 \mathrm{ml}$ hyperpolarised ${ }^{3} \mathrm{He}$ and $800 \mathrm{ml} \mathrm{N} 2$ was inhaled from a state of relaxed expiration, and ${ }^{3} \mathrm{He}$ images were acquired during the breath-hold. A $3 \mathrm{D}$ coronal spoiled gradient echo sequence ${ }^{16}$ with full lung coverage was used for imaging with the following parameters: field of view $35 \mathrm{~cm}^{2}$, in-plane matrix $64 \times 32,16 \times 20 \mathrm{~mm}$ slices, flip angle $1^{\circ}$, band width $62 \mathrm{kHz}$, echo time $0.8 \mathrm{~ms}$ and repetition time $2.5 \mathrm{~ms}$. This 3D volume was acquired at six time-points during the single breath-hold; at 0, 1.3, 5.8, 10.3, 14.8 and $19.3 \mathrm{~s}$.

The time-course images were analysed for any ventilation defects present in the first time-point image, which subsequently filled with gas during the breath-hold. A pattern of gas filling with gas moving gradually from the edges of the defect towards the centre was identified as being suggestive of collateral ventilation.

\section{RESULTS}

The patients with COPD scanned (five male, five female) had a mean age of 60.6 years, a mean smoking history of 39.5 pack years and a mean FEV1\% predicted of $49.6 \%$. Ventilation defects with delayed filling were seen in eight of the 10 patients with
COPD scanned (table 1). These examples varied in defect size, number and fill-rate from the most visibly striking case shown in figure 2 to much more subtle effects such as those seen in patient 3 in figure 3 . Fifteen slow-filling ventilation defects were identified, eight of these were seen to fill from the edge(s) of the defect and seven were too small to determine the gas fill pattern.

Figure $2 \mathrm{~A}-\mathrm{F}$ shows a single coronal image slice acquired from one of the patients at the six time-points. Two initially nonventilated defects, which gradually filled with ${ }^{3} \mathrm{He}$ over the timecourse of the breath-hold are highlighted with arrows. The largest defect is magnified in the top row. The images are all displayed with the same colour scale, the magnitude of which is proportional to the density of ${ }^{3} \mathrm{He}$ in that pixel. The hyperpolarised ${ }^{3} \mathrm{He} M R$ signal is non-renewable and diminishes over time owing to both the imaging procedure and natural $T_{1}$ relaxation processes due to the presence of oxygen. ${ }^{16}$ In normally ventilated regions of the lung this expected signal decay over time is observed and has been used to infer lung oxygen partial pressure. ${ }^{17}$ However, in the regions indicated the signal increases over time, with a progressive influx of polarised gas from the edge of the defects towards the centre. Sagittal reformats through the right lung at time-points $t=0 \mathrm{~s}(\mathrm{G})$ and $t=19.3 \mathrm{~s}(\mathrm{H})$ illustrate the $3 \mathrm{D}$ nature of the upper lobe ventilation defect and the related wash-in of gas. The delay to signal onset map (figure 2I) shows the time taken in seconds from the start of the data acquisition until gas arrived at each pixel. The gas-filling pattern from both of the defect edges towards the centre is not consistent with the defects being ventilated via their feeding bronchi. This suggests that gas is entering the defects via collateral pathways at the defect edges.

High-resolution CT images (eg, figure 2J) show that this patient has severe bilateral emphysema-in particular, pan-lobar disease affecting the right upper and lower lobes. The two ventilation defects in the right lung seen in figure 2 correspond to more severe disease than the surrounding tissue. There is no difference in lung structure discernible by high-resolution CT between the slow-ventilated upper defect and the defect in the lower right lung, which does not ventilate at all.

Figure 3 shows examples of slow-filling ventilation defects in two other patients. In one patient (top row) several wedgeshaped defects along the peripheral edge of the right lung are filled over the course of the breath-hold. In another patient (bottom row) delayed ventilation of a wedge defect on the outside edge of the left lung is seen.

An example of delayed filling of a peripheral ventilation defect is shown in figure 4 . The slow filling of the defect at the lower left lung edge, with a front of gas progressing slowly towards

Table 1 Patient demographics, lung function and delayed-filling ventilation defect information

\begin{tabular}{|c|c|c|c|c|c|c|c|c|c|c|c|c|c|}
\hline Patient & $\begin{array}{l}\text { Age } \\
\text { (years) }\end{array}$ & Sex & $\begin{array}{l}\text { Height } \\
\text { (cm) }\end{array}$ & $\begin{array}{l}\text { Weight } \\
\text { (kg) }\end{array}$ & $\begin{array}{l}\text { Smoking } \\
\text { history } \\
\text { (pack-years) }\end{array}$ & $\begin{array}{l}\text { FEV1 } \\
\text { (I) }\end{array}$ & $\begin{array}{l}\text { FEV1\% } \\
\text { predicted }\end{array}$ & $\begin{array}{l}\text { FEV1/ } \\
\text { FVC (\%) }\end{array}$ & $\begin{array}{l}\text { TLCO } \\
\text { (mmol/ } \\
\text { kPa.min) }\end{array}$ & $\begin{array}{l}\text { TLCO } \\
\text { (\% predicted) }\end{array}$ & $\begin{array}{l}\text { Number of } \\
\text { defects with } \\
\text { delayed filling }\end{array}$ & $\begin{array}{l}\text { Volumes of } \\
\text { defects }\left(\mathrm{cm}^{3}\right)\end{array}$ & $\begin{array}{l}\text { Defect fills } \\
\text { from the } \\
\text { edge(s)? }\end{array}$ \\
\hline 1 & 55 & $\mathrm{~F}$ & 155 & 62 & 30 & 0.83 & 39 & 29 & 1.8 & 25 & 2 & $173.3,81.2$ & $\mathrm{Y}, \mathrm{Y}$ \\
\hline 2 & 64 & $\mathrm{~F}$ & 160 & 97 & 50 & 0.72 & 34 & 33 & 3.7 & 51 & 4 & $16.2,15.6,2.3,0.8$ & $Y, Y, U, U$ \\
\hline 3 & 64 & $M$ & 163 & 53 & 25 & 1.50 & 56 & 34 & 3.4 & 44 & 1 & 6.6 & $Y$ \\
\hline 4 & 62 & $M$ & 183 & 98 & 35 & 1.08 & 30 & 30 & 5.0 & 49 & 2 & $46.1,65.9$ & $Y, Y$ \\
\hline 5 & 68 & $M$ & 169 & 83 & 90 & 1.54 & 55 & 49 & 3.4 & 41 & 1 & 4.9 & $U$ \\
\hline 6 & 64 & $M$ & 170 & 87 & 20 & 1.39 & 47 & 37 & 7.0 & 81 & 0 & 0 & NA \\
\hline 7 & 63 & $\mathrm{~F}$ & 157 & 70 & 50 & 0.72 & 35 & 44 & 2.5 & 36 & 2 & $15.4,4.3$ & $Y, U$ \\
\hline 8 & 58 & $M$ & 172 & 99 & 35 & 2.61 & 81 & 54 & 5.0 & 54 & 0 & 0 & NA \\
\hline 9 & 50 & $\mathrm{~F}$ & 156 & 89 & 40 & 1.69 & 74 & 62 & 5.5 & 73 & 2 & $0.9,0.7$ & $U, U$ \\
\hline 10 & 58 & $\mathrm{~F}$ & 159 & 77 & 20 & 0.99 & 45 & 45 & 3.1 & 42 & 1 & 1.5 & U \\
\hline
\end{tabular}

FEV1, forced expiratory volume in $1 \mathrm{~s}$; FVC, forced vital capacity; NA, not applicable; TLCO, Transfer Factor of the Lung for Carbon Monoxide; U, unsure (defects are too small to determine gas fill pattern); Y, yes. 


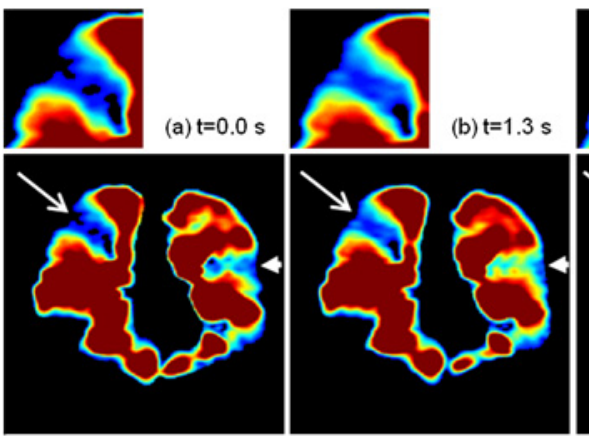

(g) Sagittal reformat, $t=0.0 \mathrm{~s}$ (h) Sagittal reformat, $t=19.3 \mathrm{~s}$
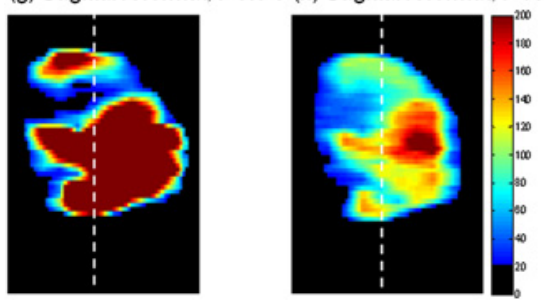

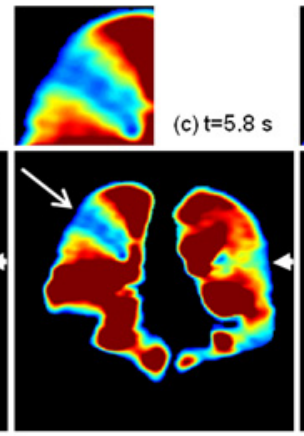

(i) Delay to signal onset

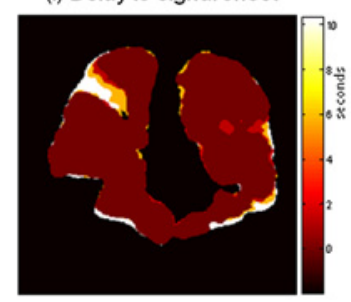

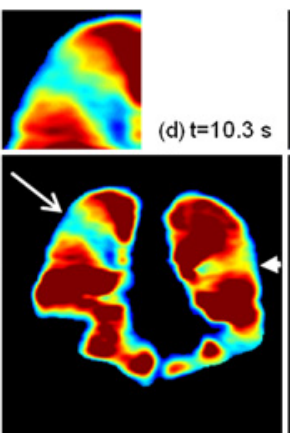

$r=1$

(e) $\mathrm{t}=14.8 \mathrm{~s}$

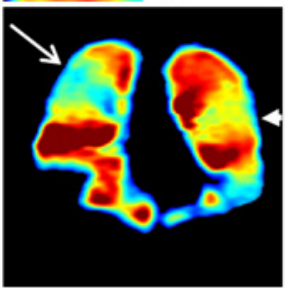

(j) HRCT image

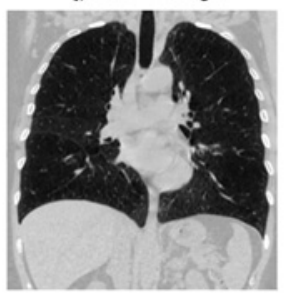

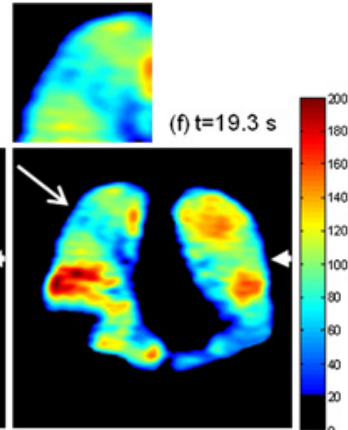

(k) Ventilation image, $t=4.2 \mathrm{~s}$

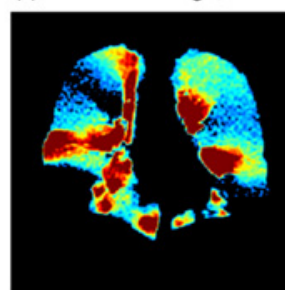

Figure 2 Images tracking collateral ventilation in a patient with chronic obstructive pulmonary disease (COPD). Images (A) to (F) show the same coronal slice at six different time points during a single breath-hold. The colour scale is the same for all images (A) to (F). The arrows indicate two collaterally ventilated defects, and the top row shows magnifications of the largest defect. (G) A sagittal reformat of the first time-point data and (H) A sagittal reformat of the last time-point data with the coronal slice position indicated with a dashed white line. (I) A delay to signal onset map for the coronal slice with time scale in seconds. (J) A high-resolution CT (HRCT) image of a similar slice. (K) A high-resolution ${ }^{3} \mathrm{He}$ MRI ventilation image of the same coronal slice acquired in the same scanning session.

the lung edge, may be due to increased resistance to air flow in the peripheral airways.

\section{DISCUSSION}

Ventilation defects with delayed filling in a pattern consistent with collateral ventilation were seen in $40 \%$ of the patients scanned. Delayed-filling ventilation defects were present in $80 \%$ of the patients but some were too small to suggest which mechanism might cause the delayed ventilation.

Delayed filling of ventilation defects may be due to collateral ventilation, partial obstruction, lung hyperinflation (air trapping), narrowing of the peripheral airways or a mixture of such mechanisms. However, in cases where the pattern of ${ }^{3} \mathrm{He}$ filling is visibly seen to progress from the defect edges towards the (a) $\mathrm{t}=0.0 \mathrm{~s}$

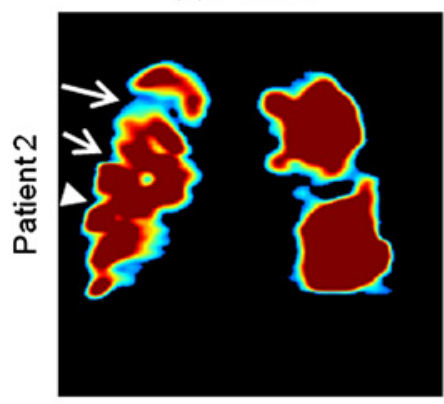

(e) $\mathrm{t}=0.0 \mathrm{~s}$

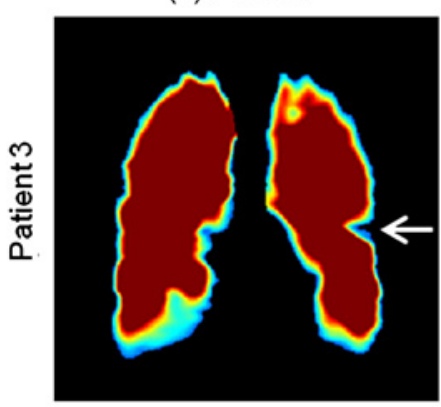

(b) $t=19.3 \mathrm{~s}$

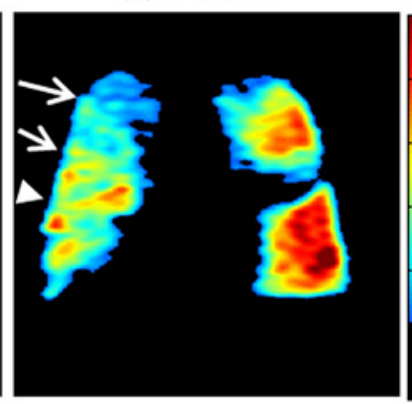

(f) $t=19.3 \mathrm{~s}$

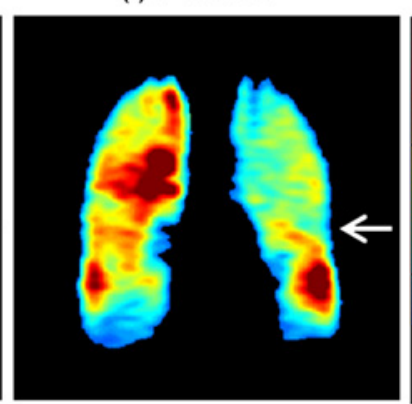

(c) Delay to signal onset

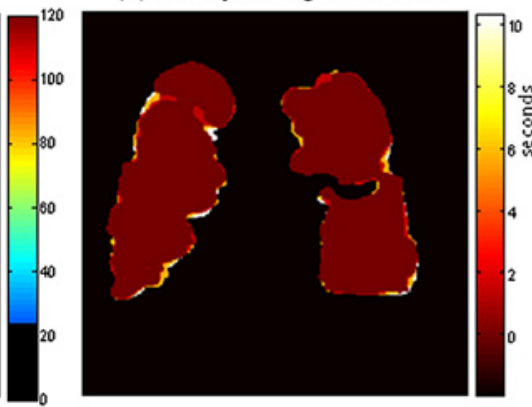

(g) Delay to signal onset

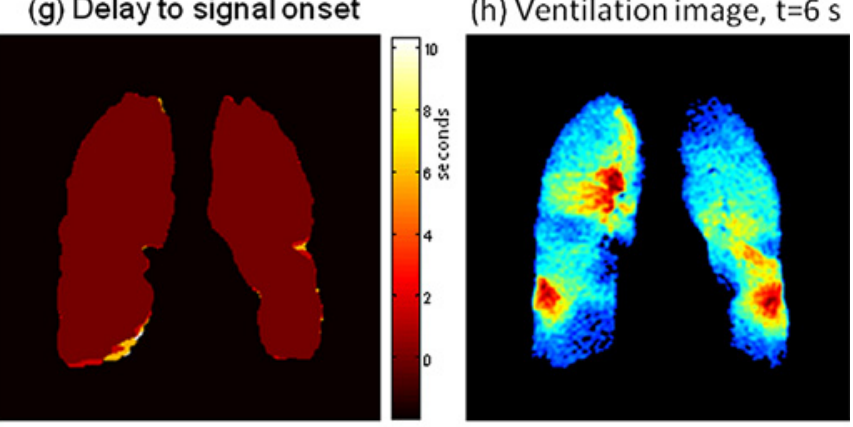

(d) Ventilation image, $\mathrm{t}=6 \mathrm{~s}$

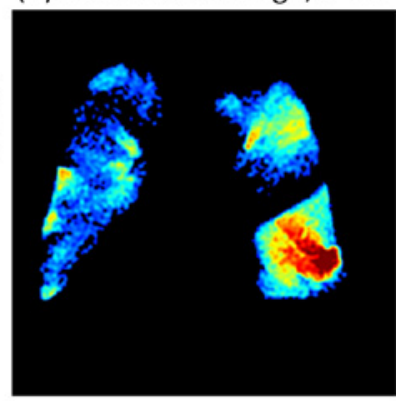

(h) Ventilation image, $t=6 \mathrm{~s}$

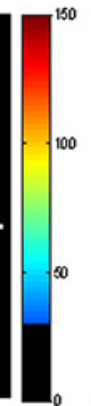

Figure 3 Images showing delayed ventilation in two other patients with chronic obstructive pulmonary disease (COPD); (top row) patient 2, (bottom row) patient 3. (A) The first time-point data. (B) The last time-point data, arrows highlight ventilation defects which fill with gas during the course of the breath-hold. (C) The delay to signal onset map. (D) A high-resolution ventilation image of the same slice. (E) to (H) show the same respectively for patient 3. 
(a) $t=0.0 \mathrm{~s}$

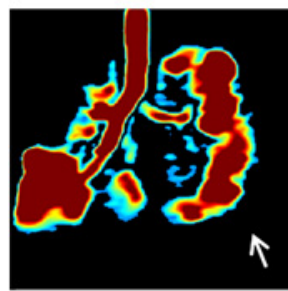

(b) $t=1.3 \mathrm{~s}$

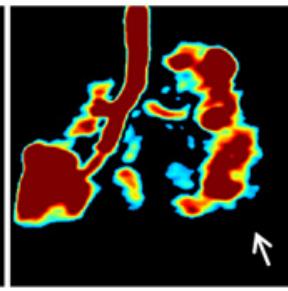

(g) Delay to signal onset

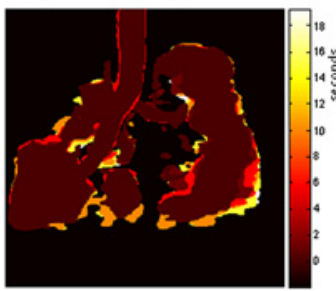

(c) $t=5.8 \mathrm{~s}$

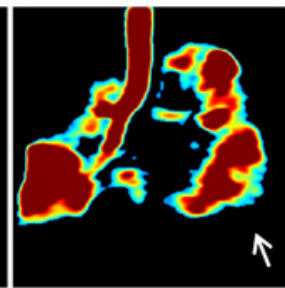

$\uparrow$ (d) $t=10.3 \mathrm{~s}$

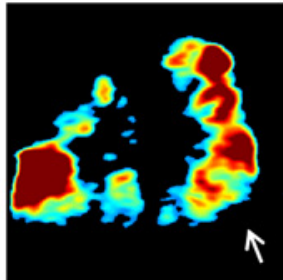

(e) $t=14.8 \mathrm{~s}$

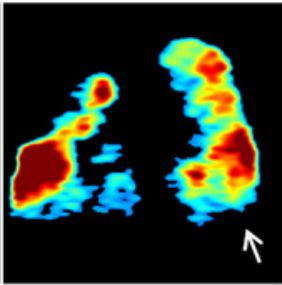

(h) Ventilation image, $t=4.2 \mathrm{~s}$
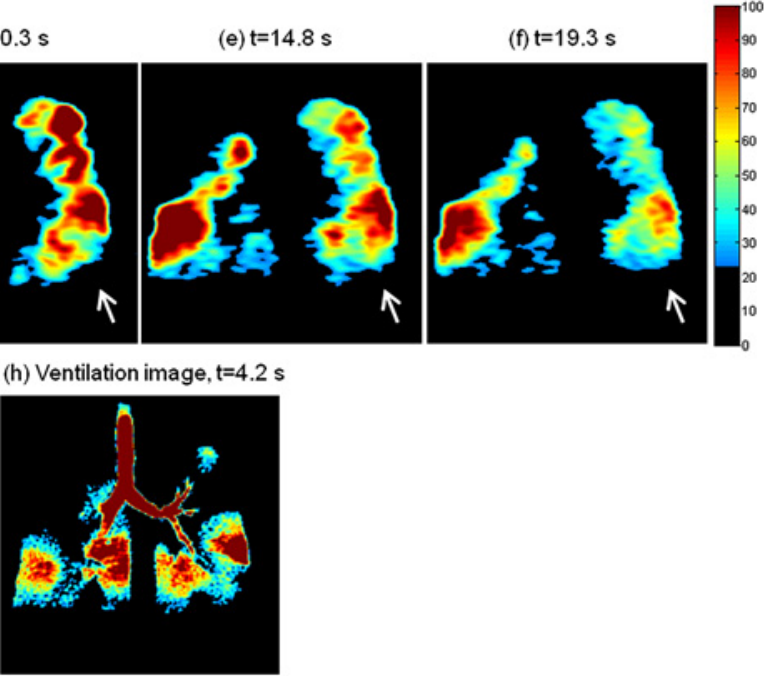

Figure 4 Images showing slow filling of a peripheral ventilation defect. Images (A) to (F) A coronal slice at six time-points during the breath-hold. (G) A delay to signal onset map and $(\mathrm{H}) \mathrm{A}$ high-resolution ventilation image of the same slice.

centre (eg, figure 2) we believe that the slow filling is due to collateral ventilation. The example shown in figure 4 with peripheral delayed filling might represent sensitivity to peripheral airways flow resistance in the "quiet zone ${ }^{18}$ of the lungs.

Collaterally ventilated defects may arise from centrilobular emphysema, ${ }^{19}$ where centrilobular spaces form in lobules with obliterated terminal bronchioles ${ }^{20}$ and may become ventilated via collateral pathways. The larger collaterally ventilated defects might be networks of lobules with interconnecting centrilobular spaces.

High-resolution ${ }^{3} \mathrm{He}$ ventilation images, which were acquired as a single time-frame snapshot during the same scanning sessions (figures $2 \mathrm{~K}, 3 \mathrm{D}, \mathrm{H}$ and $4 \mathrm{H}$ ) show no information about the collateral wash-in of gas. A whole breath-hold is needed for a single acquisition of the lung volume with high-resolution multislice imaging, meaning that there is no time-resolved aspect to the data and changes over time cannot be detected. The slice acquisition timing of a high-resolution ventilation dataset will change the appearance of defects with delayed ventilation. For example, if images of a slow-filling defect are obtained near the start of a breath-hold it will appear as a signal void (eg, figure $2 \mathrm{~K}$ ) but if images of the same defect are obtained later in the multislice acquisition, after gas has entered it, the ventilation defect will contain some signal (eg, figure $3 \mathrm{H}$ ).

The case of collateral ventilation presented here appears to be intersegmental, which is consistent with previously reported collateral ventilation in COPD detected using xenon-enhanced dual-energy $\mathrm{CT}^{21}$

By virtue of its low atomic mass ${ }^{3} \mathrm{He}$ in air is more diffusive than xenon in air or pure air alone, so it is likely to show a faster and potentially amplified effect, allowing delayed/collateral ventilation to be visualised directly over the period of a single breath-hold (within $20 \mathrm{~s}$ ). The use of this technique with hyperpolarised ${ }^{129} \mathrm{Xe}$ MRI may also allow visualisation of delayed/collateral ventilation, although the lower diffusivity of ${ }^{129} \mathrm{Xe}$ may slow the process to beyond a realistic single breath-hold time in patients.

The imaging sequence used is simple and available on all MRI systems equipped for hyperpolarised gas lung imaging. The sequence is readily adaptable, and from these initial data a breath-hold of $15 \mathrm{~s}$ with four evenly spaced data acquisition time-points should be sufficient to capture delayed-ventilation effects similar to those seen here, with the potential for an associated increased spatial resolution in the images.

In conclusion, a method is demonstrated for direct imaging of delayed ventilation within a single breath-hold, which can visualise what we believe to be collateral ventilation in COPD The technique gives $3 \mathrm{D}$ full lung coverage, allowing global assessment of delayed and collateral ventilation pathways with regional sensitivity.

Acknowledgements Steven Parnell and Peggy Xu for help with hyperpolarised gas dispensing. Thanks to Professor Jim Hogg at the University of British Columbia for useful comments on possible disease mechanisms of collateral ventilation in the review process.

Funding Funded by GlaxoSmithKline (RES111175) and UK EPSRC (EP/D070252/1). Polariser support from GE Healthcare.

\section{Competing interests None.}

Ethics approval South Yorkshire REC (REC reference: 10/H1310/11).

Provenance and peer review Not commissioned; externally peer reviewed.

\section{REFERENCES}

1. Hogg JC, Macklem PT, Thurlbeck WM. The resistance of collateral channels in excised human lungs. J Clin Invest 1969;48:421-31.

2. Cetti EJ, Moore AJ, Geddes DM. Collateral ventilation. Thorax 2006;61:371-3.

3. Voshaar TH. [Collateral ventilation] (In German). Pneumologie 2008;62:355-60.

4. Toma TP, Hopkinson NS, Hillier J, et al. Bronchoscopic volume reduction with valve implants in patients with severe emphysema. Lancet 2003;361:931-3.

5. Lausberg HF, Chino K, Patterson GA, et al. Bronchial fenestration improves expiratory flow in emphysematous human lungs. Ann Thorac Surg 2003;75:393-8.

6. Aljuri N, Freitag L. Validation and pilot clinical study of a new bronchoscopic method to measure collateral ventilation before endobronchial lung volume reduction. $J$ App/ Physiol 2009;106:774-83.

7. Higuchi T, Reed A, Oto $T$, et al. Relation of interlobar collaterals to radiological heterogeneity in severe emphysema. Thorax 2006;61:409-13.

8. Salanitri J, Kalff V, Kelly M, et al. 133Xenon ventilation scintigraphy applied to bronchoscopic lung volume reduction techniques for emphysema: relevance of interlobar collaterals. Intern Med J 2005;35:97-103.

9. Chae EJ, Seo JB, Kim N, et al. Collateral ventilation in a canine Model with bronchial obstruction: assessment with xenon-enhanced dual-energy CT. Radiology 2010;255:790-8.

10. Goo HW, Yang DH, Kim N, et al. Collateral ventilation to congenital hyperlucent lung lesions assessed on xenon-enhanced dynamic dual-energy CT: an initial experience. Korean J Radiol 2011;12:25-33.

11. Goo HW, Chae EJ, Seo JB, et al. Xenon ventilation CT using a dual-source dualenergy technique: dynamic ventilation abnormality in a child with bronchial atresia. Pediatr Radiol 2008;38:1113-16.

12. Owers-Bradley JR, Fichele $S$, Bennattayalah $A$, et al. MR tagging of human lungs using hyperpolarized 3He gas. J Magn Reson Imaging 2003;17:142-6. 
13. Woods JC, Yablonskiy DA, Chino K, et al. Magnetization tagging decay to measure long-range (3)He diffusion in healthy and emphysematous canine lungs. Magn Reson Med 2004;51:1002-8.

14. Wang C, Altes TA, Mugler JP 3rd, et al. Assessment of the lung microstructure in patients with asthma using hyperpolarized 3He diffusion MRI at two time scales: comparison with healthy subjects and patients with COPD. J Magn Reson Imaging 2008;28:80-8.

15. Bouchiat M, Carver T. C. V. Nuclear polarization in ${ }^{3} \mathrm{He}$ gas induced by optical pumping and dipolar exchange. Phys Rev Lett 1960:5:373-5

16. Wild JM, Fichele S, Woodhouse N, et al. 3D volume-localized p02 measurement in the human lung with 3He MRI. Magn Reson Med 2005;53:1055-64.
17. Deninger AJ, Eberle $\mathrm{B}$, Ebert $\mathrm{M}$, et al. Quantification of regional intrapulmonary oxygen partial pressure evolution during apnea by (3)He MRI. J Magn Reson 1999;141:207-16.

18. Mead J. The lung's "quiet zone". N Engl J Med 1970;282:1318-19.

19. Leopold JG, Gough J. The centrilobular form of hypertrophic emphysema and its relation to chronic bronchitis. Thorax 1957;12:219-35.

20. McDonough JE, Yuan R, Suzuki M, et al. Small-airway obstruction and emphysema in chronic obstructive pulmonary disease. N Engl J Med 2011;365:1567-75.

21. Park EA, Goo JM, Park SJ, et al. Chronic obstructive pulmonary disease: quantitative and visual ventilation pattern analysis at xenon ventilation CT performed by using a dual-energy technique. Radiology 2010;256:985-97.

\section{Journal club}

\section{Endothelial cells as regulators of cytokine storms during influenza infection}

The morbidity and mortality of severe influenza infections has been attributed to excessive inflammatory cytokine production and early innate immune cell recruitment. This study highlights the pulmonary endothelium as a central regulator of the cytokine storm, in particular demonstrating the role of the sphingosine-1-phosphate (S1P1) signalling system.

S1P1 receptor specific agonists were shown to significantly reduce cytokine responses and innate inflammation following infection of mice with mouse adapted influenza virus. Similar results were found using mice infected with a virulent isolate of the H1N1 pandemic influenza virus. S1P1 agonism also led to a reduction in mouse mortality.

Using an eGFP-S1P1 receptor knockin mouse and flow cytometry, high levels of S1P1 expression were detected in lung lymphatic and vascular endothelium, CD4 T cells and B cells. However, S1P1 agonist treatment of knockin mice did not alter the expression levels suggesting an effect through functional activation rather than receptor degradation. Furthermore, S1P1 treatment of virus infected lymphocyte deficient mice inhibited the innate inflammatory response, proposing pulmonary endothelium cells as the main regulators of this process. LysM-GFP mice were employed to demonstrate that S1P1 mediated inhibition of cellular infiltration occurs as a result of cytokine downregulation. However, cytokine production and cell recruitment were shown to be distinct events.

Using a variety of chemical and genetic methods, this study provides data to support the pulmonary endothelium as a key orchestrator of the cytokine storm. Manipulation of the cellular signalling pathways involved may have broad therapeutic implications.

Teijaro JR, Walsh KB, Cahalan S, et al. Endothelial cells are central orchestrators of cytokine amplification during influenza virus infection. Cell 2011;146:980-91.

\section{Gautam}

Correspondence to Dr M Gautam, ST6 Respiratory Medicine, University Hospital of Aintree, Liverpool, UK; manish16gautam@gmail.com

Provenance and peer review Not commissioned; internally peer reviewed.

Published Online First 14 December 2012

Thorax 2012;67:617. doi:10.1136/thoraxjnl-2011-201437 